\title{
Nudos críticos de procesos gerenciales en unidades productivas de Cuyes de engorde en Milagro, Ecuador
}

\author{
Critical nodes of management processes in productive units of Guinea Pigs fattening in \\ Milagro, Ecuador
}

\author{
Alex Castro-García1 iD y Juan Carlos Nava* iD \\ ${ }^{1}$ Universidad Agraria del Ecuador, Guayaquil, Ecuador. ${ }^{2}$ Universidad del Zulia,Maracaibo, Venezuela. \\ Correo Electrónico: navauniversidaddelzulia@gmail.com
}

\begin{abstract}
RESUMEN
Este estudio propone identificar los nudos críticos (NC) en los procesos gerenciales (PG) en unidades productivas (UP) de Cuyes de Engorde (CE) en Milagro, Provincia de Guayas, Ecuador, siendo la cría de $\mathrm{C}$ una actividad de importancia en diferentes zonas rurales. Por lo tanto, se visitó la mencionada zona, aplicando un cuestionario socio-económico como instrumento de recolección de datos; la población y la muestra estuvieron constituidas por 260 Productores $(P)$ de CE. La investigación fue de carácter explicativa, según el diseño no experimental, de campo. Los NC que limitaron los PG en las UP de CE fueron: deficiente toma de decisiones (DTD), ausencia del presupuesto (AP), no llevan registros (NR) y no programan las actividades a realizar (NPA). Los $\mathrm{P}$ al identificar los NC deberían analizar cuáles son los factores que inciden en la producción de $\mathrm{CE}$; planificando, revisando que necesidades existen, realizado ajustes, utilizando registros, con la necesidad de mejorar el manejo, la productividad y los ingresos netos; tomando en cuenta los costos de producción, con la elaboración del presupuesto, con supervisión y ajustes. Pese a que los $P$ están realizando diferentes actividades relacionadas con los CE y las consideraron importantes, se detectó un desconocimiento en definir estrategias para luego tomar decisiones en beneficio de la UP, de acuerdo a cada situación real que se presente, con estrategias definidas previamente para buscar obtener mejores rendimientos.
\end{abstract}

Palabras clave: Nudos críticos; procesos gerenciales; cuyes de engorde

\begin{abstract}
This study proposes to identify the critical nodes $(C N)$ in the management processes (MP) in productive units (PU) of Guinea Pig Fattening (GPF) in Milagro, Guayas Province, Ecuador. Being GPF an important activity in different rural areas, therefore, the mentioned zone was visited, applying a socio-economic questionnaire as a data collection instrument; the population and the sample consisted of 260 producers (P) of GPF. The research was explanatory in nature, according to the non-experimental field design. The CN that limited the MP in the PU of GPF were poor decision making, absence of the budget, they do not keep records and do not schedule the activities to be carried out. P, when identifying $\mathrm{CN}$, should analyze the factors that affect the production of GPF; planning, reviewing what needs exist, making adjustments, using records, with the need to improve management, productivity and net income; taking into account production costs, with the preparation of the budget, with supervision and adjustments. Despite the fact that $P$ are carrying out different activities related to GPF and considered them important, there was a lack of knowledge in defining strategies to later make decisions for the benefit of the PU, according to each real situation that arises, with previously defined strategies, to seek to obtain better returns.
\end{abstract}

Key words: Critical nodes; management processes; guinea pigs for fattening 


\section{INTRODUCCIÓN}

El Cuy (C) (Cavia porcellus L.), comúnmente conocido como Guinea Pig, cobayo, conejillo de indias, cuyo, acure, ente otros; es un mamífero roedor que en edad adulta puede medir entre 20 a 25 centímetros $(\mathrm{cm})$ de largo y pesar entre 0,5 y 1,5 kilogramos $(\mathrm{kg})$, los machos son de mayor tamaño que las hembras [1]. Es considerado un mamífero originario de la región andina de Sudamérica, tiene hábitos nocturnos, es inofensivo, nervioso y sensible al frío [4].

El $C$ es un pequeño roedor, utilizado como alimento en diferentes países. Su crianza es generalizada en el ámbito rural como un animal de carne para autoconsumo, constituyéndose en una excelente alternativa para diversificar la dieta de las familias [20]. Existen varias razas de $\mathrm{C}$, obtenidas de diversos cruces para obtener un mejoramiento genético. En el caso de Ecuador, aún persiste la crianza de $\mathrm{C}$ criollos, a nivel de indígenas y campesinos. Igual, se presenta el sistema familiar, cuando el destino de la producción es para autoconsumo; el sistema familiar - comercial, cuando los excedentes de la producción se destinan a la venta y el sistema comercial, cuando la producción tiene la finalidad principal de la venta [8].

Se considera que, al tecnificar la crianza del C, con una mejor calidad de alimentación, se puede mejorar el peso de los animales en menor tiempo; el manejo de la población de $\mathrm{C}$ permite lograr un índice productivo de comercialización a las nueve semanas (sem) y una conversión alimentaria (CA) con alimentación mixta. La alimentación es un aspecto importante en la crianza de $\mathrm{C}$, ya que de esto podría depender el rendimiento y calidad de los animales [13]. Tradicionalmente, los $\mathrm{C}$ criollos han sido alimentados con forraje, pero con los trabajos de mejoramiento genético y de nutrición, los nuevos genotipos presentan requerimientos nutricionales superiores para optimizar los rendimientos en crecimiento y reproducción [5]. Así mismo, la cría de $\mathrm{C}$ es una actividad pecuaria importante en las zonas rurales de Perú, Ecuador, Bolivia y en menor proporción en Colombia, Argentina, Chile y Venezuela [12].

En este contexto, se entiende por nudos críticos (NC) una situación que perjudica el desarrollo eficiente de una organización en el logro de sus objetivos y metas, traduciéndose en que sean poco eficientes [21]. De esta manera, para Centanaro y Nava [7] se debería conocer si los productores manejan información, cuentan con planes escritos, fijan metas, objetivos, estrategias, manejan un presupuesto conociendo los ingresos y costos totales, realizan seguimiento y control, entre otros.

Por lo tanto, es de gran importancia que los productores logren identificar los NC en los procesos gerenciales (PG) del manejo de $\mathrm{CE}$, teniendo en cuenta que disminuyen la calidad, competitividad y productividad de las unidades de productivas (UP). Se deben identificar los NC en todo el proceso productivo, buscando lograr una alta productividad, mayor eficiencia, efectividad, gran capacidad competitiva y excelente calidad. En este sentido, el objetivo de la investigación consistió en identificar los NC que existen en los PG en UP de CE en Milagro, provincia del Guayas, Ecuador.

\section{MATERIALES Y MÉTODOS}

\section{Descripción de la zona de estudio}

Milagro, provincia del Guayas se encuentra a 45 kilómetros $(\mathrm{km})$ de Guayaquil, tiene una de superficie $226,08 \mathrm{~km}^{2}$. Se localiza en las coordenadas $2^{\circ} 08^{\prime} 05^{\prime \prime} \mathrm{S} \mid 79^{\circ} 35^{\prime} 14^{\prime \prime} \mathrm{O}$. La altitud predominante es de 8 y 15 metros $(\mathrm{m})$ sobre el nivel del mar (msnm). Su temperatura promedio anual está alrededor de los $25^{\circ} \mathrm{C}$ y su precipitación es de 1.361 milímetros $\cdot a_{n ̃ o}^{-1}\left(\mathrm{~mm} \cdot \mathrm{a}^{-1}\right)$ [10].

\section{Tipo y diseño de la investigación}

La investigación fue de carácter explicativa; se trató de revelar el cómo y el por qué ocurre el fenómeno y se dan argumentaciones teóricas, lógicas, históricas y causales de los hechos [9], identificando los NC en el PG en las UP de C en Milagro, provincia del Guayas. Según el diseño fue no experimental, de campo; Arias [3] estableció que la investigación de campo consiste en la recolección de datos directamente de los sujetos investigados, o de la realidad donde ocurrieron los hechos; el investigador obtiene la información pero no altera las condiciones existentes.

\section{Población y muestra}

El universo de estudio estuvo constituido por los productores (P) de C de Milagro, provincia del Guayas. Para determinar la población se establecieron los siguientes criterios: UP destinadas principalmente a la producción de C, UP con más de un a de estar establecidas, UP con actividad productiva, $\mathrm{P}$ con personalidad jurídica o natural pero que no tuvieran identificación gubernamental. Luego de realizar las respectivas visitas se delimitó el área de estudio, con un total de 260 UP de C (260 P), siendo esta la población y la muestra.

\section{Técnicas e instrumentos de investigación}

Se realizaron visitas preliminares a la mencionada zona, con el propósito de efectuar el primer contacto con los $\mathrm{P}$ y lograr un primer acercamiento. Se diseñó y aplicó un cuestionario socioeconómico como instrumento de recolección de datos, aplicado de manera individual a los $\mathrm{P}$ de $\mathrm{C}$ que conformaron la población y la muestra, constituido por preguntas cerradas que luego fueron tabuladas y analizadas.

\section{Validez y confiabilidad del instrumento}

Se consultó la opinión de cinco expertos relacionados con el área de estudio, quienes revisaron el instrumento y aportaron opiniones y sugerencias utilizadas para la construcción definitiva de dicho instrumento. Luego se realizó una prueba piloto a $10 \mathrm{P}$ de $C$, para definir si el instrumento planteado estaba acorde con la capacidad de interpretación de los mencionados $P$.

\section{Procesamiento y análisis de la información}

Una vez aplicado el instrumento de recolección de datos, se procedió a realizar el procesamiento de los datos y el análisis de la información a través de distribución de frecuencias, para ello se utilizó el paquete estadístico Statistical Analysis System [22]. Se obtuvo información de los NC que existieron en los PG del manejo de C en Milagro, provincia del Guayas. La descripción de los indicadores desde el punto de vista cualitativo, permitió la elaboración del diagrama de causa-efecto o diagrama de Ishikawa [11]. 


\section{RESULTADOS Y DISCUSIÓN}

\section{Identificación de los NC de los PG}

Luego de realizar las respectivas visitas y aplicar el instrumento de recolección de datos, se obtuvo la información necesaria. Los resultados podrían apoyar a que se mejoren los $\mathrm{PG}$ con soluciones prácticas y efectivas para lograr cambios positivos. Por lo tanto, se detectaron cualitativamente los NC de los PG, a través de la construcción del diagrama de causa-efecto (FIG. 1).

Los NC que limitaron el PG en las UP de $C$ fueron: deficiente toma de decisiones (DTD), ausencia de presupuesto (AP), no llevan registros (NR) y no programan las actividades a realizar (NPA). Los $\mathrm{P}$ al identificar los NC deberían analizar cuáles son los factores que inciden en la producción de C; con la necesidad de mejorar la productividad y los ingresos netos.

\section{Deficiente toma de decisiones (DTD)}

El 82,69\% de los $P(215)$, realizaron una deficiente DTD, sin definición de estrategias, bajos rendimientos, ejecución improvisada, con un lento crecimiento y engorde de los C, entre otros (FIG. 2).

En este sentido, se dificultó establecer cuál fue el estado de ganancias y pérdidas, en detrimento de la toma de decisiones, planificación y evaluación de la actividad productiva. Se presentó un desconocimiento de los $\mathrm{P}$ en definir estrategias para luego tomar decisiones en beneficio de la UP, para mejorar el crecimiento y engorde de los C. Según López [13] en Ecuador, la mayoría de UP de $\mathrm{C}$ se manejan de manera poco técnica, con animales con bajo peso al sacrificio, tiempos prolongados de crianza, ligado a la escasez de alimentos en determinadas épocas del a, repercutiendo en el desarrollo normal del C.

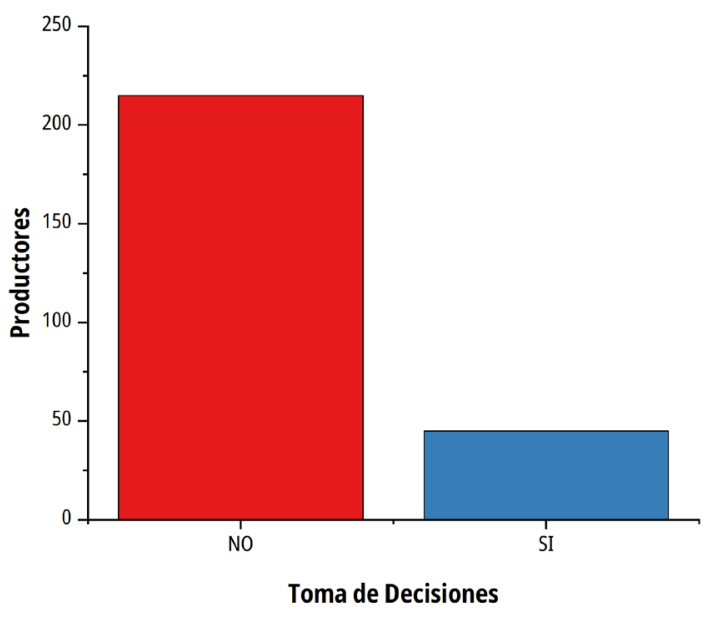

FIGURA 2. Deficiente toma de decisiones en unidades productivas de Cuyes de engorde en Milagro, Provincia del Guayas, Ecuador

En la toma de decisiones TDD, debe haber conocimiento y determinación en lo que se está decidiendo; se realiza en todos los ámbitos, donde la comunicación es de gran importancia para establecer los objetivos, definir estrategias, entre otros; los factores que pueden tener mayor incidencia y afectación en la TDD son los económicos, normativos, personales, sociales, políticos, físicos, entre otros [23].

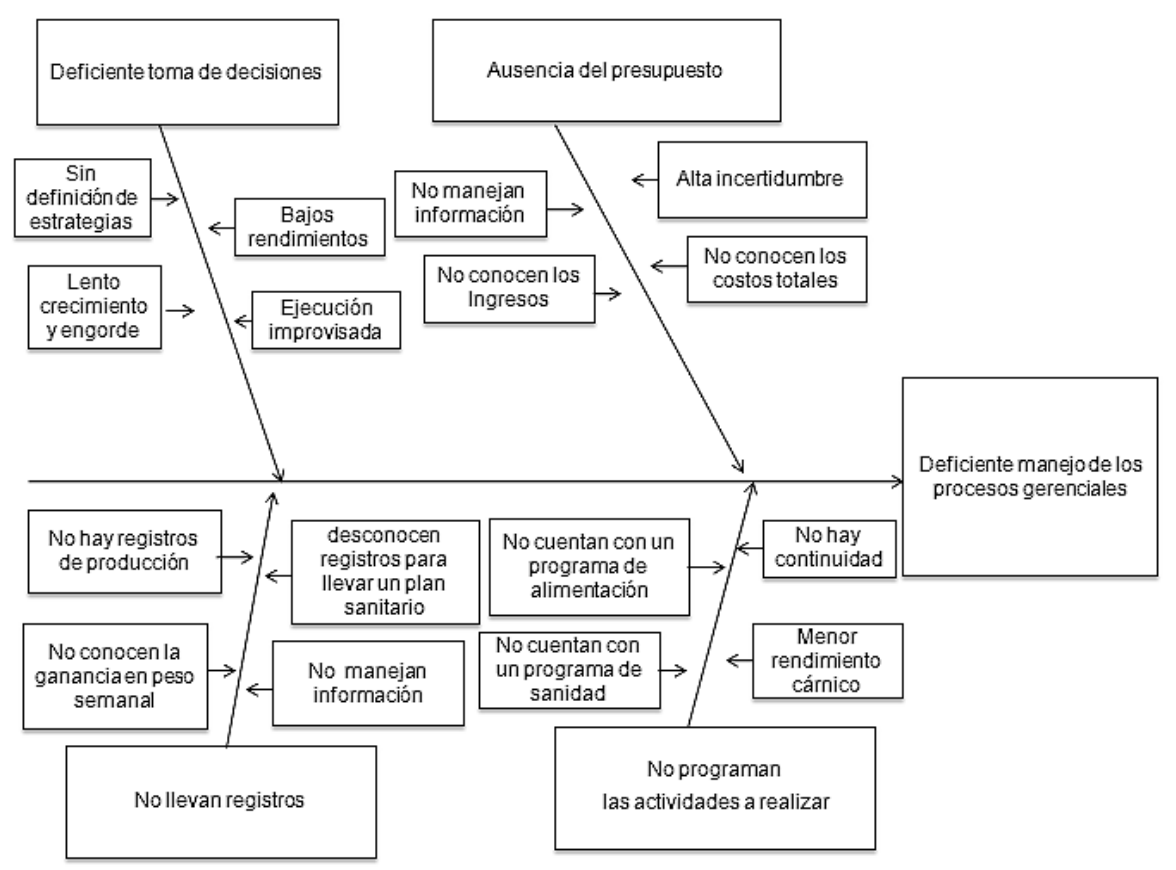

FIGURA 1. Diagrama Causa-Efecto para detectar nudos críticos de las unidades productivas de Cuyes de engorde en Milagro, Provincia del Guayas, Ecuador 
Por lo tanto, la TDD se debería fundamentar en antecedentes básicos concretos, para lograr tomar las mejores decisiones posibles, teniendo por supuesto los objetivos y las metas bien definidos. La TDD debería tener su punto de partida en la planificación, revisando las limitaciones que se podrían presentar, organizando, integrando, supervisando, realizando seguimiento y control apropiado en cada una de las UP de C. Pese a que los $P$ están realizando diferentes actividades relacionadas con los $C$ y las consideraron importantes, es necesario manejar muy bien todos los aspectos relacionados con la TDD para cada UP, de acuerdo a cada situación real que se presente, con estrategias definidas previamente, buscando obtener mejores rendimientos.

\section{Ausencia del presupuesto (AP)}

El $95,76 \%$ de los $\mathrm{P}$ (249) no realizaron un presupuesto, no conocen los ingresos, costos totales, no manejan información, presentándose una alta incertidumbre (FIG. 3).

En cada UP de $\mathrm{C}$ se requiere de la realización oportuna de una serie de prácticas, tendientes a mantener la producción de la misma, a niveles de productividad por encima del umbral económico aceptable, pero es de gran necesidad que exista un presupuesto previamente elaborado, buscando que los $P$ sean cada día (d) más competitivos, generando cambios que se adapten a sus UP para poder mantenerse en el tiempo siendo exitosos y no solamente conozcan cuánto dinero perciben en una sem o en un mes, sino que adviertan también cuanto fueron los ingresos totales, costos totales, entre otros.

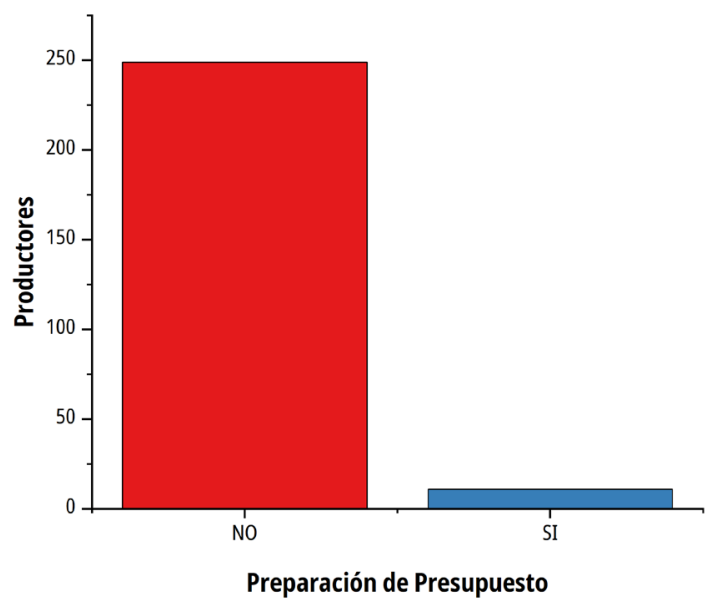

FIGURA 3. Ausencia del presupuesto en unidades productivas de Cuyes de engorde en Milagro, Provincia del Guayas, Ecuador

\section{No llevan registros (NR)}

De acuerdo con los resultados obtenidos, se encontró que el $93,07 \%$ de los $P$ (242), no utiliza registros, solo el 6,93 \% de los $P$ (18) si llevó registros en su UP; donde se evidencia, que no hay registros de producción, no manejan información, desconocen registros para llevar un plan sanitario, no conocen la ganancia en peso (GdP) semanal de los C, entre otros (FIG. 4).

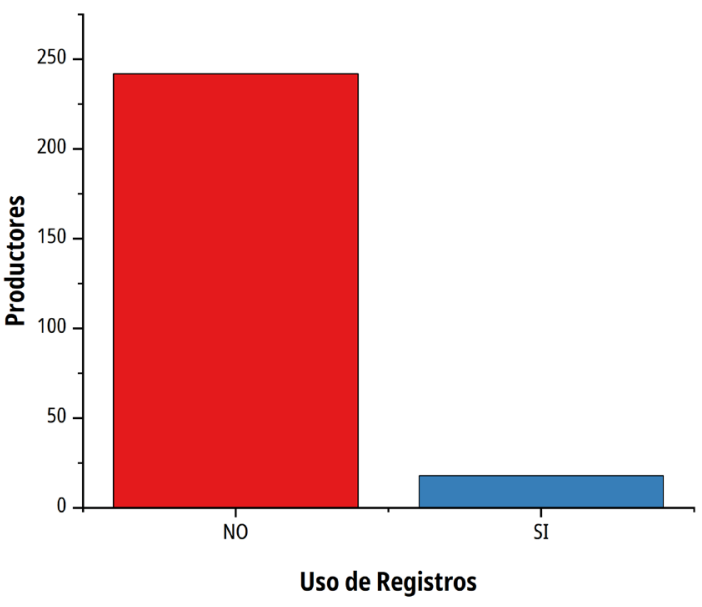

FIGURA 4. Uso de registro por parte de Productores en unidades productivas de Cuyes de engorde en Milagro, Provincia del Guayas, Ecuador

Al respecto, Silva y col. [21] indicaron, que la mayoría de los $P$ utiliza registros manuales sin información sistematizada que permita evaluar el ejercicio económico. Además, se encontró que la forma, el número, tipo de registro y las personas involucradas en el proceso no presentan los mecanismos más adecuados para llevar registros. En este contexto, Nava y col. [18] señalaron que al llevar registros, los $\mathrm{P}$ podrían trabajar en forma más ordenada y eficiente al programar sus actividades, con un plan de acción que ayude a solucionar problemas, y aprovechar las oportunidades que se presenten.

En el sistema de manejo de $\mathrm{C}$, es útil manejar registros para mantener el control de los animales, los cuales permiten conocer los aspectos de producción de mayor interés. Se debe tomar en cuenta la fecha del empadre (inicio de la etapa reproductiva) y de los partos, conocer el número de partos por año, tamaño de camada, conocer y llevar registros de las GdP, consumo de materia seca, CA, relación entre incremento de peso y CA registrado, entre otros [6]; para Lozada y col. [14] se debe conocer cómo mejorar significativamente la GdP y la CA, así como los costos de producción, relación beneficio costo, edad óptima económica de beneficio, entre otros.

Por lo tanto, es de gran importancia unificar criterios de manejo por parte de los $\mathrm{P}$, en relación al peso de los $\mathrm{C}$ y la GdP semanal, con una vinculación de los involucrados, con recomendaciones y situaciones deseadas para mejorar la producción de los $\mathrm{C}$, como una actividad pecuaria tan relevante en las zonas rurales.

\section{No programan las actividades a realizar (NPA)}

El 88,07\% de los $\mathrm{P}$ (229) no realizaron planificación de las actividades a realizar en las UP, solo el $11,93 \%$ de los $P$ si realizó la planificación (31 P), por lo que se refleja que no cuentan con un programa de sanidad, no cuentan con un programa de alimentación, no existiendo continuidad, con un menor rendimiento cárnico, entre otros (FIG. 5). 


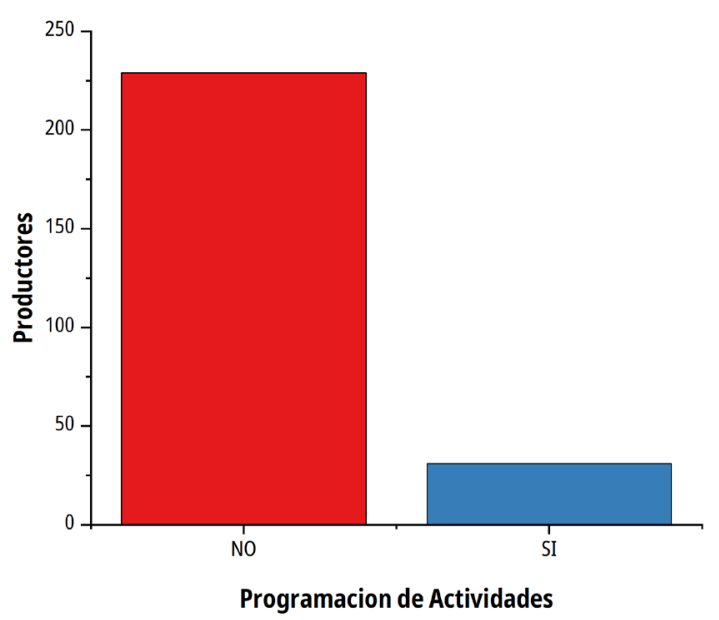

FIGURA 5. Programacion de actividades por parte de Productores en unidades productivas de Cuyes de engorde en Milagro, Provincia del Guayas, Ecuador

La aplicación de la función planificación se caracterizó por una deficiente ejecución. Hubo un grado importante de $\mathrm{P}$ de $\mathrm{C}$ que no realizaron actividades fundamentales para la planificación operativa en las UP. Como parte de la planificación se deberían fijar metas y objetivos, realizando un manejo apropiado de los problemas, tomando las decisiones, considerando los resultados obtenidos en las producciones anteriores. Se debería realizar la planificación de actividades por escrito, tomando en cuenta la productividad, ingresos, costos, entre otros; cómo se va a trabajar la UP durante un tiempo determinado, que incluya el monitoreo y evaluación de la misma.

Siendo de gran importancia que los $\mathrm{P}$ de $\mathrm{C}$ planifiquen todas las actividades a realizar, que utilicen herramientas eficientes de planificación y puedan conocer los resultados del proceso en un determinado periodo, poder evaluar y efectuar los correctivos en aquellos aspectos que estén incidiendo en la obtención de los mejores resultados. Los $\mathrm{P}$ planificaron algunas actividades, pero no consideraron realizar dicha planificación de forma escrita, de manera que pudieran asegurar el logro de los objetivos; realizando la ejecución improvisada de actividades que serían trascendentales por la necesidad de las mismas en un momento determinado en el manejo de los $\mathrm{C}$.

Según Silva y col. [21] se observan procesos de planificación con deficiencia en los criterios empresariales para definir la misión del negocio, el cortoplacismo de las metas, el hecho de no planificar por escrito, y con un tiempo de antelación muy corto, la ausencia de presupuestos y cronogramas de actividades. En este sentido, para Meza y col. [16] en la cría de C se descuidan aspectos importantes como una adecuada alimentación, manejo, higiene, entre otros, dando lugar a que no se alcancen buenos índices reproductivos y productivos. La nutrición juega un rol importante en una UP. El conocimiento de los requerimientos nutritivos de los $C$ permitirá poder elaborar raciones balanceadas que logren satisfacer las necesidades en cada una de las fases biológicas por las que atraviesa (gestación, lactancia, engorde).
En este contexto, también se deben llevar registros del programa de alimentación; Masaquiza y col. [15] indicaron, que es muy importante conocer los requerimientos nutricionales que deben estar presentes en la dieta alimenticia diaria para que los $C$ puedan desarrollarse y reproducirse con normalidad. Según Lozada y col. [14] debe existir una suplementación desde antes del inicio del empadre para mejorar la fertilidad, tamaño de camada, índice de productividad, entre otros. Se deberían utilizar diferentes alternativas con alto potencial productivo y alto valor nutritivo para mejorar la alimentación, intentando no incrementar los costos de producción [19].

Por lo tanto, al programar las actividades, se debe realizar el programa de alimentación, siendo uno de los aspectos más importante, ya que una alimentación insuficiente en calidad y cantidad, trae como consecuencia una serie de trastornos. Todos los $\mathrm{P}$ señalaron la importancia de la alimentación, pero sin un programa establecido; conocer los requerimientos nutritivos de los $C$ podría permitir elaborar raciones balanceadas que logren satisfacer las necesidades de mantenimiento, crecimiento y producción.

En este sentido, Andrade y col. [2] señalaron, que es importante la disponibilidad de los nutrientes y la frecuencia, para lograr los rendimientos productivos de esta especie. Para Lozada y col. [14] se deben mejorar los sistemas de alimentación de los animales como forma de obtener un mayor rendimiento cárnico y la reducción de los costos de producción. Los ensayos realizados se orientan a la alimentación de $\mathrm{C}$ con balanceados formulados con la inclusión de ingredientes alternativos [17].

Finalmente, identificados los NC, se deberían establecer los objetivos, las estrategias y las acciones a seguir. Siendo urgente establecer planes de desarrollo, que busquen mejorar la calidad de vida, con una relación y complemento entre los involucrados, para que mejoren con base a sus propios recursos y capacidades, que se organicen y que participen en la toma de decisiones.

\section{CONCLUSIONES}

Los NC que limitaron el PG en las UP de $C$ fueron DTD, AP, NR, y NPA.

Los $\mathrm{P}$ al identificar los NC deberían analizar cuáles son los factores que inciden en la producción de $\mathrm{C}$; planificando, revisando que necesidades existen, realizado ajustes, utilizando registros, con la necesidad de mejorar el manejo de los $\mathrm{C}$, la productividad y los ingresos netos; tomando en cuenta los costos de producción, con la elaboración del presupuesto, con supervisión y ajustes.

Se presentó un desconocimiento de los $\mathrm{P}$ en definir estrategias para luego tomar decisiones en beneficio de la UP, de acuerdo a cada situación real que se presente, con estrategias definidas previamente.

\section{REFERENCIAS BIBLIOGRÁFICAS}

[1] ALIAGA, L.; MONCAYO, R.; RICO, E.; CAYCEDO, A. Producción de cuyes. Aspectos generales de los cuyes. Fondo Editorial de la Universidad Católica Sedes Sapientiae. Lima, Perú. 808 pp. 2009.

[2] ANDRADE, V.; FUENTES, I.; VARGAS, J.; LIMA, R.; JÁCOME, A. Alimentación de cuyes en crecimiento-ceba a base de gramíneas tropicales adaptadas a la Región Amazónica. Rev. Electr. Vet. 17(1): 1-7. 2016. 
[3] ARIAS, F. El proyecto de investigación. Introducción a la Metodología Científica. $6^{a}$ Ed. Ed. Episteme. Caracas, Venezuela. 143 pp. 2012.

[4] AVILÉS, D.; MARTÍNEZ, A.; LANDI, V.; DELGADO, J. El cuy (Cavia porcellus): un recurso andino de interés agroalimentario. Anim. Genet. Res. 55: 87-91. 2014.

[5] CAMINO, J.; HIDALGO, V. Evaluación de dos genotipos de cuyes (Cavia porcellus) alimentados con concentrado y exclusión de forraje verde. Rev. Invest. Vet. Perú. 25(2): 190-197. 2014.

[6] CARBAJAL, C. Evaluación preliminar de tres alimentos balanceados para cuyes (Cavia porcellus) en acabado en el valle del Mantaro. Universidad Agraria La Molina. Lima, Perú. Trabajo de Grado. 78 pp. 2015.

[7] CENTANARO, P.; NAVA, J. Nudos críticos de procesos gerenciales en unidades productivas de banano, Milagro, Ecuador. Rev. CEA. 7(13): e1554. 2021.

[8] CRUZ, M. Comportamiento productivo de progenies F2 de cuatro cruzamientos entre grupos raciales de cuyes (Cavia porcellus) de hembras F1 con machos macabeo y peruano mejorado. Tumbaco-Pichincha. Universidad Central del Ecuador. Trabajo de Grado. 80 pp. 2013.

[9] HERNÁNDEZ, R.; FERNÁNDEZ, C.; BAPTISTA, L. Metodología de la investigación. Definición del tipo de investigación. Editorial McGraw-Hill Interamericana. México. 152 pp. 2010.

[10] INSTITUTO NACIONAL DE METEROLOGÍA E HIDROLOGÍA. (INAMHI). Boletín Agroclimático Decadal informativo. Litoral ecuatoriano, situación climatologica y perspectivas. No. DEI-BAD-30-2018. Quito. 42 pp. 2018.

[11] ISIKAWA, K. ¿Qué es el control total de la calidad? Editorial Norma. Venezuela. 232 pp. 2003.

[12] JURADO, H.; CABRERA, E.; SALAZAR, J. Comparación de dos tipos de sacrificio y diferentes tiempos de maduración sobre variables físico-químicas y microbiológicas de la carne de cuy (Cavia porcellus). Rev. Invest. Med. Vet. Zoot. 63(3): 201-217. 2016.

[13] LÓPEZ, M. Evaluación de tres sistemas de alimentación sobre el rendimiento productivo en cuyes de la línea inti, andina y Perú. Universidad Técnica de Ambato. Cevallos, Ecuador. Trabajo de Grado. 91 pp. 2016.
[14] LOZADA, P.; JIMÉNEZ, R.; SAN MARTÍN, F.; HUAMÁN, A. Efecto de la inclusión de cebada grano y semilla de girasol en una dieta basada en forraje sobre el momento óptimo de beneficio de cuyes. Rev. Invest. Vet. Perú. 24: 25-31. 2013.

[15] MASAQUIZA, M.; DIEGO, A.; PEREDA, J.; CURBELO, L.; FIGUEREDO, R.; CERVANTES, M. Intensificación de los sistemas agropecuarios y su relación con la productividad y eficiencia. Resultados con su aplicación. Rev. Prod. Anim. 29(2): 57-64. 2017.

[16] MEZA, G.; CABRERA, R.; MORÁN, J.; MEZA, F.; CABRERA, C.; MEZA, C.; MEZA, J.; CABANILLA, M.; LÓPEZ, F.; PINCAY, J.; BOHÓRQUEZ, T.; ORTIZ, J. Mejora de engorde de cuyes (Cavia porcellus L.) a base de gramíneas y forrajeras arbustivas tropicales en la zona de Quevedo, Ecuador. IDESIA, Chile. 32(3):75-80. 2014.

[17] MORALES, A. Evaluación de dos niveles de energía en el comportamiento productivo de cuyes (Cavia porcellus) de la raza Perú. Rev. Invest. Vet. Perú. 22: 177-182. 2011.

[18] NAVA, J.; SÁNCHEZ, A.; ORTEGA, J. Gestión de planificación económica en el cultivo del plátano en el estado Zulia, Venezuela. Rev. Fac. Agron. LUZ. 34: 371-396. 2017.

[19] RODRÍGUEZ, I.; GUEVARA, E. Producción de materia seca y valor nutritivo de la leguminosa arbustiva Cratylia argentea en el sur del estado Anzoátegui, Venezuela. Rev. Fac. Cien. Vet. UCV. 2: 589-594. 2002.

[20] SÁNCHEZ, A.; SÁNCHEZ, S.; GODOY, S.; DIAZ, R.; VEGA, N. Gramíneas tropicales en el engorde de cuyes mejorados sexados (Cavia porcellus) en la zona de la Maná. Rev. Cien. Tec. Ecuador. 2: 25-28. 2009.

[21] SILVA, D.; RINCÓN, N.; PÉREZ, Y.; PEÑA, M.; URDANETA, $F$. Nudos críticos de procesos gerenciales en unidades de producción de ganadería de doble propósito del municipio Machiques de Perijá, estado Zulia. Rev. Fac. Agron. LUZ. 24(3): 547-576. 2007.

[22] STATISTICAL ANALISYS SYSTEM INSTITUTE. SAS/STAT User's guide, Rel. 9.1.3 Cary, NC. 2014.

[23] URDANETA, K. Gestión de proyectos en consejos comunales del municipio Maracaibo. Universidad del Zulia. Facultad de Ciencias Económicas y Sociales. División de estudios para Graduados. Maracaibo, Venezuela. Tesis doctoral. Venezuela. 423 pp. 2010. 